\title{
Synthesis of quinoxalines in the presence of heteropoly acids
}

\author{
Fatemeh Hakimi $^{{ }^{*}}$ and Bi Bi Fatemeh Mirjalili ${ }^{\mathbf{b}}$
}

${ }^{a}$ Department of Chemistry, Payamenoor University, PO BOX 19395-3697 Tehran, Iran, Yazd, Rezvanshahr, Sadoogh ${ }^{b}$ Department of Chemistry, College of Science, Yazd University, Yazd, P.O.Box 89158-13149, I. R. Iran

CHR ON I L E ABSTRACT

Article history:

Received October 25, 2012

Received in Revised form

December 6, 2012

Accepted 19 January 2013

Available online

19 January 2013

Keywords:

Quinoxalines

1,2-Diketones

o-Phenylenediamines

Keggin-type Heteropolyacids

\begin{abstract}
Efficient synthesis of quinoxaline derivatives from the reaction of $\alpha$-diketones and $o$ phenylenediamines in the presence of Keggin-type heteropolyacids (HPA) such as $\mathrm{H}_{3} \mathrm{PMo}_{12} \mathrm{O}_{40}, \mathrm{H}_{4} \mathrm{SiW}_{12} \mathrm{O}_{40}, \mathrm{~K}_{7} \mathrm{PMo}_{2} \mathrm{~W}_{9} \mathrm{O}_{40}, \mathrm{H}_{3} \mathrm{PW}_{12} \mathrm{O}_{40} \cdot \mathrm{SiO}_{2}$ and $\mathrm{H}_{3} \mathrm{PW}_{12} \mathrm{O}_{40}$ in high yields and short reaction times, and at room temperature is introduced.
\end{abstract}

\section{Introduction}

Heteropoly acids as solid acid catalysts are green catalysts with respect to their non-corrosive nature, safety, low quantity of waste and easy separation. One of the unique features that make solid heteropoly acids economically and environmentally attractive is their stability and high acidity.

Quinoxalines are important heterocycles in medicinal chemistry ${ }^{1,2}$ and have biological activities such as antibacterial and anti-inflammatory activities ${ }^{3,4}$. The best reported method for synthesis of quinoxalines is the reaction of aryl 1,2-diamines with a 1,2-dicarbonyl compounds in the presence of an acid as catalyst. Acetic acid ${ }^{5}$, iodine ${ }^{6}, \mathrm{CuSO}_{4} \cdot 5 \mathrm{H}_{2} \mathrm{O}^{7}, \mathrm{Zn}[(\mathrm{L}) \text {-proline }]^{8}$, Ni-nanoparticles ${ }^{9}$, gallium(III)triflate ${ }^{10}$, montmorillonite $\mathrm{K}_{10}{ }^{11}$, task-specific ionic liquids ${ }^{12}, \mathrm{MnCl}_{2}{ }^{13}$, and $\mathrm{ZrO}_{2} / \mathrm{Ga}_{2} \mathrm{O}_{3} / \mathrm{MCM}-41^{14}$ and alumina-supported heteropolyoxometalates ${ }^{15}$ have been applied in the above mentioned method.

* Corresponding author.

E-mail addresses: fatemeh.hakimi@yahoo.com (F. Hakimi)

C 2013 Growing Science Ltd. All rights reserved.

doi: $10.5267 /$ j.ccl.2013.01.001 


\section{Result and Discussions}

In continuation of our investigations on the applications of solid acids in organic synthesis, we have investigated the synthesis of quinoxalines in the presence of heteropoly acid at room temperature. Herein, we report that heteropoly acids are efficient catalysts for the synthesis of quinoxaline derivatives comparable with some other applied catalysts. The reaction of 1,2phenylenediamine with benzil was investigated for optimization of the reaction conditions. Reaction at different temperatures and various molar ratios of substrates in the presence of heteropoly acid revealed that the best results were obtained under solvent-free conditions at room temperature and a molar ratio of 1,2-phenylenediamine: benzil: heteropoly acid equal to 1:1:0.01.

Various 1,2-phenylenediamines and 1,2-diketones were used as substrates for the synthesis of quinoxalines under solvent free at room temperature (Scheme 1 and Table 1).

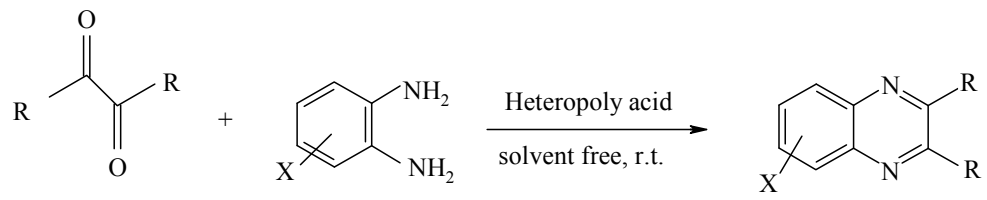

$\mathrm{R}=\mathrm{Me}, \mathrm{Ar} \quad \mathrm{X}=\mathrm{H}, 4-\mathrm{NO}_{2}$

Scheme 1. Preparation of quinoxaline derivatives in the presence of heteropolyacids

Table 1. The synthesis of quinoxaline from 1, 2-phenylenediamine (1 mmol) and benzil (1 mmol) using heteropoly acids $\left(\mathrm{A}: \mathrm{H}_{3} \mathrm{PMo}_{12} \mathrm{O}_{40}, \mathrm{~B}: \mathrm{H}_{4} \mathrm{SiW}_{12} \mathrm{O}_{40}, \mathrm{C}: \mathrm{K}_{7} \mathrm{PMo}_{2} \mathrm{~W}_{9} \mathrm{O}_{40}\right.$, D: $\mathrm{H}_{3} \mathrm{PW}_{12} \mathrm{O}_{40} \cdot \mathrm{SiO}_{2}, \mathrm{E}: \mathrm{H}_{3} \mathrm{PW}_{12} \mathrm{O}_{40}, 1$ mol \%) as catalyst.

\begin{tabular}{|c|c|c|c|c|c|c|c|c|c|}
\hline Entry & $\mathrm{R}$ & $\mathrm{X}$ & Product & \multicolumn{5}{|c|}{ Time (min)/ Yield (\%) } & M.P. ${ }^{\circ} \mathrm{C}$ \\
\hline & & & & $\mathrm{A}$ & $\mathrm{B}$ & $\mathrm{C}$ & $\mathrm{D}$ & $\mathrm{E}$ & \\
\hline 1 & $\mathrm{Ph}$ & $\mathrm{H}$ & & $3 / 98$ & $3 / 98$ & 2/99 & $2 / 95$ & $10 / 90$ & $127-128$ \\
\hline 2 & $\mathrm{Ph}$ & $\mathrm{NO}_{2}$ & & $2 / 99$ & $3 / 98$ & $3 / 98$ & $2 / 98$ & $10 / 89$ & $192-193$ \\
\hline 3 & $4-\mathrm{OCH}_{3} \mathrm{Ph}$ & $\mathrm{H}$ & & $2 / 99$ & $3 / 98$ & $3 / 98$ & $2 / 99$ & $12 / 88$ & $152-153$ \\
\hline 4 & $4-\mathrm{OCH}_{3} \mathrm{Ph}$ & $\mathrm{NO}_{2}$ & & $3 / 99$ & $3 / 98$ & $2 / 99$ & $2 / 99$ & $10 / 88$ & 193-194 \\
\hline 5 & $\mathrm{CH}_{3}$ & $\mathrm{H}$ & & $3 / 98$ & $3 / 99$ & $3 / 98$ & $2 / 99$ & $14 / 90$ & $135-136$ \\
\hline 6 & $\mathrm{CH}_{3}$ & $\mathrm{NO}_{2}$ & & 2/99 & $3 / 99$ & $3 / 98$ & $2 / 98$ & $10 / 91$ & $185-186$ \\
\hline
\end{tabular}

A: $\mathrm{H}_{3} \mathrm{PMo}_{12} \mathrm{O}_{40}, \mathrm{~B}: \mathrm{H}_{4} \mathrm{SiW}_{12} \mathrm{O}_{40}, \mathrm{C}: \mathrm{K}_{7} \mathrm{PMo}_{2} \mathrm{~W}_{9} \mathrm{O}_{40}, \mathrm{D}: \mathrm{H}_{3} \mathrm{PW}_{12} \mathrm{O}_{40} \cdot \mathrm{SiO}_{2}, \mathrm{E}: \mathrm{H}_{3} \mathrm{PW}_{12} \mathrm{O}_{40}$ 


\section{Conclusions}

Herein, we have reported a mild, easy applicable and efficient method for the preparation of quinoxalines from benzils and $o$-phenylenediamines using small amount of heteropolyacids as highly efficient solid catalysts. These reactions are characterized by good yields and short reaction times.

\section{Acknowledgements}

The Research Council of Payamenoor University is gratefully acknowledged for the financial support for this work.

\section{Experimental}

\section{Chemicals and apparatus}

All chemicals were purchased from commercial suppliers and were used as received. All products were identified by their spectra and physical data. Melting points were measured by using the capillary tube method with an electrothermal 9100 apparatus. Polyoxometalates were prepared according to literature procedures ${ }^{16}$. The IR spectra were recorded on a Shimadzu DT-40 model 883 IR Spectrophotometer (KBr pellets, Nujol mulls, 4000-400 $\mathrm{cm}^{-1}$ ). ${ }^{1} \mathrm{H}$ NMR spectra were recorded on a Bruker- Avance DRX 400 spectrometer using TMS as an internal standard. Elemental analyses were done by Costech ECS 4010 CHNS-O analyzer.

\section{Preparation of quinoxalines catalyzed using heteropoly acids:}

A mixture of $o$-phenylenediamine $(1 \mathrm{mmol}), 1,2$-dicarbonyl $(1 \mathrm{mmol})$ and catalyst $(1 \mathrm{~mol} \%)$ was grounded in a mortar for 2-4 minute. The progress of the reaction was monitored by TLC. After completion of the reaction, the mixture was dissolved in $\mathrm{CH}_{2} \mathrm{Cl}_{2}$, filtered and washed with diethyl ether $(5 \mathrm{~mL})$ to isolate the catalyst. The solvent was evaporated under reduced pressure and the pure product was obtained.

\section{Selected spectroscopic data}

2,3-Diphenylquinoxaline (Table 1, entry 1), FT- IR: $\bar{v}(\mathrm{KBr})=3055,1541,1348,1053,771,697 \mathrm{~cm}$

${ }^{1},{ }^{1} \mathrm{H}-\mathrm{NMR}\left(400 \mathrm{MHz}, \mathrm{CDCl}_{3}\right) \delta=8.01(\mathrm{dd}, J=6.3$ and $3.6 \mathrm{~Hz}, 2 \mathrm{H}), 7.79$ (dd, $J=6.3$ and $3.4 \mathrm{~Hz}$, 2H), $7.5(\mathrm{~m}, 4 \mathrm{H}), 7.39(\mathrm{~m}, 6 \mathrm{H}) \mathrm{ppm}$. Elemental analysis, Found, \%: C 85.03; H 5.11; N 9.86.

$\mathrm{C}_{20} \mathrm{H}_{14} \mathrm{~N}_{2}$. Calculated, \%: C 85.08; H 5.00; $\mathrm{N}$ 9.92,

2,3-Bis(4-methoxyphenyl)quinoxaline (Table 1, entry 3), FT- IR: $\bar{v}(\mathrm{KBr})=2958,2838,1606,1511$, 1461, 1393, 1347, 1287, 1243, 1172, 1027, 829, 764, $596 \mathrm{~cm}^{-1},{ }^{1} \mathrm{H}-\mathrm{NMR}\left(400 \mathrm{MHz}, \mathrm{CDCl}_{3}\right) \delta=3.65$ (s, 6H), 6.69 (d, $J=8.8 \mathrm{~Hz}, 4 \mathrm{H}), 7.30$ (d, $J=8.8 \mathrm{~Hz}, 4 \mathrm{H}), 7.54$ (dd, $J=6.2$ and $3.2 \mathrm{~Hz}, 2 \mathrm{H}), 7.95$ (dd, $J=6.2$ and $3.2 \mathrm{~Hz}, 2 \mathrm{H}) \mathrm{ppm}$. Elemental analysis. Found, \%: C 77.07; H 5.15;.N 8.26; O 9.52. $\mathrm{C}_{22} \mathrm{H}_{18} \mathrm{~N}_{2} \mathrm{O}_{2}$. Calculated, \%: C 77.17; H 5.30; N 8.18; O 9.35.

2,3-Dimethylquinoxaline (Table 1, entry 5), FT-IR: $\bar{v}(\mathrm{KBr})=2923,1568,1489,1437,1363,1317$, $1164,762 \mathrm{~cm}^{-1},{ }^{1} \mathrm{H}-\mathrm{NMR}\left(400 \mathrm{MHz}, \mathrm{CDCl}_{3}\right) \delta=2.55(\mathrm{~s}, 6 \mathrm{H}), 7.77(\mathrm{dd}, J=8.8$ and $2.5 \mathrm{~Hz}, 1 \mathrm{H}), 7.79$ (dd, $J=8.8$ and $2.4 \mathrm{~Hz}, 1 \mathrm{H}) \mathrm{ppm} .{ }^{13} \mathrm{C}-\mathrm{NMR}\left(100 \mathrm{MHz}, \mathrm{CDCl}_{3}\right) \delta=23.54,30.09,128.69,129.21$, 141.45, 153.85 ppm. Elemental analysis, Found, \%: C 75.97; H 6.27; N 17.76. $\mathrm{C}_{10} \mathrm{H}_{10} \mathrm{~N}_{2}$. Calculated, \%: C 75.92; H 6.37; N 17.71.

2,3-Dimethyl-6-nitro-quinoxaline (Table 1, entry 6), FT- IR: $\bar{v}(\mathrm{KBr})=3057,2923,1616,1579$, $1525,1342,1164,743 \mathrm{~cm}^{-1},{ }^{1} \mathrm{H}-\mathrm{NMR}\left(500 \mathrm{MHz}, \mathrm{CDCl}_{3}\right) \delta=2.57(\mathrm{~s}, 3 \mathrm{H}), 2.60(\mathrm{~s}, 3 \mathrm{H}), 7.92(\mathrm{~d}, J=9.2$ $\mathrm{Hz}, 1 \mathrm{H}), 8.25(\mathrm{~d}, J=8.8 \mathrm{~Hz}, 1 \mathrm{H}), 8.70(\mathrm{~s}, 1 \mathrm{H}) \mathrm{ppm},{ }^{13} \mathrm{C}-\mathrm{NMR}\left(125 \mathrm{MHz}, \mathrm{CDCl}_{3}\right) \delta=23.72,23.93$, 
$122.75,125.29,130.34,140.34,144.13,147.58,156.69,157.62$ ppm. Elemental analysis, Found, \%: C 59.15; H 4.41; N 20.71; O 15.73. $\mathrm{C}_{10} \mathrm{H}_{9} \mathrm{~N}_{3} \mathrm{O}_{2}$. Calculated, \%: C 59.11; H 4.46; N 20.68; O 15.75.

\section{References}

1 Jaso, A.; Zarranz, B.; Aldana, I.; Monge, A. (2005), Synthesis of new quinoxaline-2-carboxylate 1,4-dioxide derivatives as anti-mycobacterium tuberculosis agents, J. Med. Chem. 48, 2019-2025, PMID: 15771444.

2 Carta, A.; Paglietti, G.; Nikookar, M.E.R.; Sanna, P.; Sechi, L. Zanetti, S. (2002) Novel substituted quinoxaline 1,4-dioxides with in vitro antimycobacterial and anticandida activity, Eur. J. Med. Chem. 37, 355-366. PMID: 12008050.

3 He, W.; Meyers, M.R.; Hanney, B.; Spada, A.; G.; Blider, H.; Galzeinski, D.; Amin, S.; Eedle, K.; Page, Jayyosi, Z.; Perrone, H. (2003), Potent quinoxaline-based inhibitors of PDGF receptor tyrosine kinase activity. part 2: the synthesis and biological activities of RPR127963 an orally bioavailable inhibitor, Bioorg. Med. Chem. Lett. 13, 3097-3100, doi:10.1016/S0960894X(03)00655-3

4 Kim,Y. B.; Kim,Y. H.; Park, J.Y.; Kim, S. K. (2004) Synthesis and biological activity of new quinoxaline antibiotics of echinomycin analogues, Bioorg. Med. Chem. Lett. 14, 541-544, doi:10.1016/j.bmcl.2003.09.086

5 Zhao, Z.; Wisnoski, D. D.; Wolkenberg, S. E.; Leister, W. H.; Wang,Y.; Lindsley, C.W. (2004) General microwave-assisted protocols for the expedient synthesis of quinoxalines and heterocyclic pyrazines Tetrahedron Lett. 45, 4873-4876, doi:10.1016/j.tetlet.2004.04.144.

6 More, S.V.; Sastry, M.N.V.; Wang, C.C.; Yao, C. (2005) Molecular iodine: a powerful catalyst for the easy and efficient synthesis of quinoxalines, Tetrahedron Lett. 46, 6345-6348, doi:10.1016/j.tetlet.2005.07.026

7 Heravi, M. M.; Taheri, S.; Bakhtiari, K.; Oskooie, H. A. (2007) On water: A practical and efficient synthesis of quinoxaline derivatives catalyzed by $\mathrm{CuSO}_{4} .5 \mathrm{H}_{2} \mathrm{O}$, Catal. Commun. 8, 211214, doi:10.1016/j.catcom.2006.06.013.

8 Heravi, M. M.; Taheri, S.; Bakhtiari, K.; Oskooie, H. A. (2007) Zn[(L)proline]: A powerful catalyst for the very fast synthesis of quinoxaline derivatives at room temperature Catal. Commun. 8, 1341-1344, doi:10.1016/j.catcom.2006.11.026.

9 Kumar, A.; kumar, S.; Saxena, A.; De, A.; Mozumdar, S. (2008), Ni-nanoparticles: an efficient catalyst for the synthesis of quinoxalines, Catal. Commun. 9, 778, doi:10.1016/j.catcom.2007.08.021.

10 Cai, J.J.; Zou, J.P.; Pan, X.Q.; Zhang, W. (2008) Gallium (III) triflate-catalyzed synthesis of quinoxaline derivatives, Tetrahedron Lett. 49, 7386-7390, doi:10.1016/j.tetlet.2008.10.058.

11 Huang, T.K.; Wang, R.; Shi, L.; Lu, X.X. (2008) Montmorillonite K-10: An efficient and reusable catalyst for the synthesis of quinoxaline derivatives in water, Catal. Commun. 9, 11431147, doi:10.1016/j.catcom.2007.10.024.

12 Dong, F.; Kai, G.; Zhenghao, F.; Xinli, Z.; Zuliang, L. (2008) A practical and efficient synthesis of quinoxaline derivative catalyzed by task-specific ionic liquid, Catal. Commun. 9, 317-320, doi:10.1016/j.catcom.2007.07.003.

13 Heravi, M. M.; Bakhtiari, K.; Oskooie, H.A.; Taheri, S. (2008) $\mathrm{MnCl}_{2}$-promoted synthesis of quinoxaline derivatives at room temperature, Heteroatom. Chem. 19, 218-220. doi 10.1002/hc. 20401 .

14 Ajaikumar, S.; Pandurangan, A. (2009) Efficient synthesis of quinoxaline derivatives over $\mathrm{ZrO}_{2} / \mathrm{M}_{x} \mathrm{O}_{y}(\mathrm{M}=\mathrm{Al}, \mathrm{Ga}$, In and $\mathrm{La})$ mixed metal oxides supported on MCM-41 mesoporous molecular sieves, Applied Catalysis A: General, 357, 184-192. doi:10.1016/j.apcata.2009.01.021.

15 Diego Ruiz, D.; Autino, J. C.; Quaranta, N.; Vazquez, P.; Romanelli, G. (2012) An efficient protocol for the synthesis of quinoxaline derivatives at room temperature using recyclable alumina-supported heteropolyoxometalates, Scientific Word J., 1-8. doi: 10.1100/2012/174784.

16 Pope, M.T. (1983), Heteropoly and isopoly oxometalates. Washington D.C. 20057 USA. 